\title{
A 12-month prospective study to evaluate the efficacy of using the treat-and-extend regimen with intravitreal aflibercept as a Second- Line Treatment for Diabetic Macular Oedema (the TADI Study)
}

\author{
Liran Tiosano (1) ${ }^{1} \cdot$ Rita Ehrlich $^{2,3} \cdot$ Yoreh Barak $^{4} \cdot$ Haya Katz $^{5} \cdot$ Russell Pokroy $^{6} \cdot$ Tarek Jaouni $^{1} \cdot$ Jaime Levy (i) $^{1}$ \\ Joel Hanhart ${ }^{7} \cdot$ Ori Segal $^{3,8} \cdot$ Shiri Shulman ${ }^{3,9} \cdot$ Michaella Goldstein $^{3,9} \cdot$ Itay Chowers $^{1}$
}

Received: 29 May 2019 / Revised: 14 April 2020 / Accepted: 15 April 2020 / Published online: 29 April 2020

(c) The Author(s), under exclusive licence to The Royal College of Ophthalmologists 2020

\begin{abstract}
Purpose To evaluate the efficacy of intravitreal aflibercept as a second-line therapy in eyes with persistent diabetic macular oedema (DMO) despite receiving initial bevacizumab treatment.

Methods A prospective multicentre study was conducted in nine academic clinics in Israel. Starting from the first follow-up visit, a treat-and-extend regimen was applied in which the treatment intervals were extended by 2 weeks based on macular thickness using SD-OCT. The primary outcome was central subfield thickness (CST) at week 52.

Results Forty-four patients ( $n=48$ eyes) were recruited to the study, and 43 eyes completed 52 weeks of follow-up. Patients received a mean $( \pm$ SD) of $7.9 \pm 3.5$ bevacizumab injections before enrolment. The mean $( \pm$ SD) CST under aflibercept therapy decreased from $468 \pm 131 \mu \mathrm{m}$ at baseline to $303 \pm 67 \mu \mathrm{m}$ at 52 weeks $(p=0.002)$, and best corrected visual acuity improved from $64 \pm 15$ ETDRS letters at baseline to $75 \pm 8$ letters at week $52(p=0.001)$. Twenty $(46 \%)$ eyes met the treatand-extend criteria and received a mean $( \pm \mathrm{SD})$ of $10.9 \pm 2$ aflibercept injections.

Conclusions Eyes with persistent DMO following initial bevacizumab therapy had a marked reduction in macular thickness and improved visual acuity following 1 year of treatment with intravitreal aflibercept. Less than half of the patients met eligibility criteria for extension of the treatment interval; for these patients, the treat-and-extend regimen resulted in a maximum treatment interval of 10 weeks during the first year.
\end{abstract}

\section{Introduction}

Diabetic retinopathy is the leading cause of vision loss in the working-age population, and diabetic macular oedema (DMO) is the most common consequence of diabetic retinopathy. DMO currently affects $\sim 750,000$ individuals in the United States alone [1-3], and intravitreal injection of

Itay Chowers

chowers@ hadassah.org.il

1 Department of Ophthalmology, Hadassah-Hebrew University Medical Center, and the Hebrew University - Hadassah School of Medicine, Jerusalem, Israel

2 Department of Ophthalmology, Rabin Medical Center, Petah Tikva, Israel

3 Sackler Faculty of Medicine, Tel Aviv University, Tel Aviv, Israel

4 Department of Ophthalmology, Rambam Medical Center, Haifa, Israel compounds that target vascular endothelial growth factor (VEGF) is currently considered the first-line treatment for the majority of DMO cases [4-10].

The Protocol T clinical trial of the Diabetic Retinopathy Clinical Research Network (www.DRCR.net) found that the recombinant fusion protein aflibercept provided superior results compared with the monoclonal full length anti-VEGF

5 Department of Ophthalmology, Kaplan Medical Center, Rehovot, Israel

6 Department of Ophthalmology, Assaf Harofeh Medical Center, Be'er Ya'akov, Israel

7 Department of Ophthalmology, Shaare Zedek Medical Center, Jerusalem, Israel

8 Department of Ophthalmology, Meir Medical Center, Kfar Saba, Israel

9 Department of Ophthalmology, Tel Aviv Medical Center, Tel Aviv, Israel 
antibody bevacizumab, and the monoclonal anti-VEGF antibody fragment ranibizumab in a sub-group of patients with DMO who presented with poor visual acuity. Among this sub-group of patients with poor visual acuity, the superiority of aflibercept over ranibizumab, noted at 1 year, was no longer identified and aflibercept maintained it superiority only compared with bevacizumab after 2 years of follow up [1113]. On the other hand, aflibercept, bevacizumab and ranibizumab, yielded similar results in patients who presented with higher visual acuity [14]. Furthermore, unlicensed bevacizumab is more cost-effective than both aflibercept and ranibizumab. Thus, in our country bevacizumab is often used as the first-line anti-VEGF treatment for DMO [12].

Despite the favourable response to first-line anti-VEGF therapy, up to $60 \%$ of eyes treated using the abovementioned protocols have some degree of persistent macular oedema; this persistent oedema is slightly more prevalent, and visual outcome is generally worse, in eyes treated with bevacizumab [12, 15]. Moreover, several studies, most of which are retrospective, have shown that switching to a different anti-VEGF drug may provide improved anatomical and/or functional outcome [16-22].

Several treatment algorithms are currently used for the timing of anti-VEGF treatments in DMO. Clinical trials often use either a fixed monthly regimen or a pro re nata (PRN) approach to treat DMO, resulting in anatomical and functional improvements that are maintained for $>3$ years $[8,23,24]$. Disadvantages of the fixed monthly treatment algorithm include substantial treatment-related burdens for the patient, the healthcare system and society. Thus the fixed monthly regimen is not commonly used in the clinic $[10,23]$. Following monthly injections, and once the patient's DMO has stabilised, switching of a PRN regimen may reduce the number of injections required compared with continuing with the fixed monthly injection schedule; the benefit of such a treatment schedule in DMO was documented, in the Protocol I clinical trial [5]. The advantage of this approach is that the eyes could achieve an initial gain in visual acuity, followed by stable outcome, with a substantial reduction in the number of injections and visits over time. However, frequent follow-up visits to exclude the recurrence of DMO are still required when using this algorithm, particularly early after switching to the PRN-based treatment regimen.

In contrast to the approach described above, the treat-andextend regimen (TER), which was originally introduced for managing age-related macular degeneration, allows for an incremental increase in treatment intervals, with the aim of identifying the longest possible interval for a given patient. This algorithm has several advantages, including a reduced frequency of clinic visits and a proactive-rather than reactive-approach, with potential benefits in terms of visual outcome [25-29]. The RETAIN and TREX-DME studies prospectively examined the management of DMO using ranibizumab, with a loading dose of 3-5 monthly injections followed by TER [30, 31]. The TER arms in both studies showed non-inferior visual outcome compared with the fixed monthly regimen group in the TREX-DME study and compared with the PRN group in the RETAIN study [31]. Moreover, both studies found a marked reduction in treatment burden in the TER groups [31, 32]. It is important to note that even with a significant reduction in macular thickness and improved visual acuity following the first injection, TER studies often include this initial loading dose of at least 3-5 monthly injections.

Although large prospective clinical trials demonstrate the efficacy of using aflibercept as a first-line therapy for DMO $[8,12,13,23]$, relatively limited prospective data are available regarding the use of aflibercept as a second-line therapy in DMO following an incomplete response to firstline bevacizumab. In addition, use of a modified second-line TER approach in DMO has not been reported previously. Thus, to investigate further the efficacy of using aflibercept as a second-line therapy combined with TER (without a loading dose), we conducted a multicentre prospective clinical trial in DMO patients who responded poorly to prior first-line bevacizumab treatment.

\section{Materials and methods}

\section{Participants and study design}

The TADI (Treat-and-Extend Regimen of Aflibercept $2 \mathrm{mg}$ / $0.05 \mathrm{ml}$ as a Second-Line Treatment for Diabetic Macular Oedema) study was a 12-month prospective, multicentre, open-label, single-arm clinical trial. A total of 44 patients ( $n=48$ eyes) were enrolled from May 2016 through September 2017 at nine academic centres in Israel. The patients were then followed for 52 weeks. The study was approved by the respective institutional review board at each participating clinical centre, and all patients provided written informed consent. The TADI study was registered with ClinicalTrials.gov (identifier no. NCT02633852).

The inclusion criteria included a partial or completely absent response to first-line intravitreal injections of bevacizumab for DMO. The first-line treatment was provided in the clinic prior to recruitment in the TADI trial. Incomplete response (i.e. refractory DMO) was defined based on spectral domain optical coherence tomography (SD-OCT) criteria and included residual intraretinal fluid (IRF) and/or subretinal fluid (SRF) involving the centre of the fovea, as well as central subfield thickness (CST) of $\geq 300 \mu \mathrm{m}$ (retinal thickness including SRF and/or IRF) measured using Heidelberg OCT. In addition, eyes had to receive 4-12 bevacizumab injections prior to inclusion in the TADI 
study, and the last 3 injections had to be provided at an interval of 4-6 weeks; this interval was chosen because it reflects the interval commonly used in real-life clinic settings. In addition, the last injection had to be administered within 6 months prior to enrolment in the study, and fluid (i.e., IRF and/or SRF) had to be present at every visit (defined as refractory DMO). Finally, for inclusion in the study, the patient's study eye was required to have best corrected visual acuity (BCVA) of 20-80 letters on the Early Treatment Diabetic Retinopathy Study (ETDRS) visual acuity chart (Snellen equivalent: $20 / 20$ to 20/200).

Exclusion criteria included the presence of any ocular pathology other than DMO that could be associated with the impaired vision, including vein occlusion, pigment abnormalities and/or dense sub-foveal hard exudates. In addition, eyes with active or suspected ocular/periocular infection or inflammation, a clinically significant epiretinal membrane, vitreomacular traction, or a macular hole were excluded. Eyes with uncontrolled intraocular pressure (IOP) or any intraocular surgery or thermal laser treatment within 3 months of starting the trial were also excluded.

The primary end point was the change in CST at week 52 compared with baseline. Secondary end points included BCVA at week 52, a gain or loss of 10 ETDRS letters by week 52, and the total number of aflibercept injections during the 12-month study.

\section{Study protocol}

BCVA, OCT and fluorescein angiography (FA) were performed using standardised protocols. OCT images from the screening visit were reviewed by the principal investigators at the respective sites in order to confirm that the inclusion and exclusion criteria were met. To be considered eligible for the study, the FA had to be deemed eligible by the participating centre, and the patient's clinical findings and OCT images had to be approved by two additional PIs at other participating centres. A unanimous consensus was required in order to confirm the patient's participation in the study.

At enrolment (day 0), each patient received an intravitreal injection of aflibercept $(2 \mathrm{mg}$ in a volume of $0.05 \mathrm{ml})$, followed by subsequent injections every 4 weeks (i.e. monthly injections) as long as there was evidence of persistent fluid. Once complete resolution of the DMO was achieved, the TER was then applied. This protocol was designed to apply TER early, without the need for mandatory monthly loading doses. At the start of TER, the patient received an injection of aflibercept, and the next follow-up interval was extended by 2 weeks relative to the preceding interval; the maximum treatment interval was 4 months. If either SRF or IRF appeared at any point after the start of TER, the interval between visits and treatments was reduced to the last interval in which the macula was fluid-free. The intravitreal injections of aflibercept were discontinued after 6 initial monthly injections in cases in which there was no improvement in DMO (defined as a decrease in central macular subfield thickness of $\geq 10 \%$ compared with the previous visit and/or an increase of $\geq 1$ ETDRS lines); the patient was then followed every 4 weeks until the end of the study. In the event of an increase in macular thickness of $\geq 10 \%$ or and/or a loss of $\geq 1$ ETDRS line after treatment was discontinued, the injections were resumed. Patients were eligible for rescue treatment with a focal laser beginning in week 24. The final exam was performed in week 52 .

At each visit, a full ophthalmic examination was performed in both eyes, including BCVA and IOP assessment using Goldmann applanation tonometry, noting of any adverse events, and SD-OCT/HD-OCT using a Heidelberg Spectralis (Heidelberg Engineering, Heidelberg, Germany) or Zeiss CIRRUS (Carl Zeiss Meditec, Dublin, CA) scanner; measurements were performed using the appropriate correction factor of $10 \mu \mathrm{m}$ based on CIRRUS HD-OCT in order to compare the thickness to the Spectralis SD-OCT [33]. FA was performed at the screening visit only.

An historical retrospective control group was also used in this study. Data from this group of consecutive patients ( $n=13$ eyes) were collected from the Retina Clinic of the Hadassah Medical Center. This group of eyes had an incomplete response to bevacizumab as a first-line therapy as defined by the inclusion criteria of the TADI study, yet continued to receive monthly injections of bevacizumab for $\geq 12$ months.

\section{Statistical analysis}

Statistical analyses were performed using SPSS (version 24.0; IBM Corp., Armonk, NY). The sample size was calculated using Hochberg's procedure to control the type I error rate for the primary end point at 5\% (two-sided) and to provide $80 \%$ probability that the change in the CST would be significant. The values for BCVA and CST are reported as the mean, $\mathrm{SD}$, median and range. Because the data were normally distributed, the BCVA and CST data were compared within and between groups using an ANOVA or Student's $t$-test. Where appropriate, a sensitivity analysis was performed using the nonparametric Wilcoxon-signed rank test or chi-square test. Differences with a $p$ value $\leq 0.05$ were considered significant.

\section{Results}

\section{Baseline characteristics}

Forty-eight eyes in 44 patients were recruited into the study from nine academic medical centres in Israel. The mean 
Table 1 Demographics and baseline characteristics of the study eyes ( $n=48$ eyes in 44 patients).

\begin{tabular}{ll}
\hline Age (years) mean \pm SD & $62 \pm 9$ \\
Male/Female, number $(\%)$ & $22 / 22(50 / 50 \%)$ \\
HBA1C\%, mean \pm SD & $8.1 \pm 1.9 \%$ \\
Smoker, number (\%) & $5(11 \%)$ \\
Hypertension, number (\%) & $20(45 \%)$ \\
Hyperlipidemia, number (\%) & $26(59 \%)$ \\
Insulin use, number (\%) & $28(63 \%)$ \\
Diabetic retinopathy, number (\%) & \\
NPDR & $45(94 \%)$ \\
PDR & $3(6 \%)$ \\
Focal/grid laser, number $(\%)$ & $2(4 \%)$ \\
PRP laser, number (\%) & $2(4 \%)$ \\
Lens status, number $(\%)$ & \\
Phakic & $39(81 \%)$ \\
Pseudophakic & $9(19 \%)$ \\
Vitreoretinal interface, number $(\%)$ & \\
Attached vitreous & $28(58 \%)$ \\
PVD & $10(21 \%)$ \\
ERM & $5(10 \%)$ \\
Retinal fluid, number $(\%)$ & \\
IRF & $40(83 \%)$ \\
SRF & $10(21 \%)$ \\
\hline
\end{tabular}

Where applicable, the percentage is based on the total number of patients or the total number of eye.

$H B A 1 C$ haemoglobin A1C, NPDR non-proliferative diabetic retinopathy, $P D R$ proliferative diabetic retinopathy, $P R P$ pan-retinal photocoagulation, $P V D$ posterior vitreous detachment, $E R M$ epiretinal membrane, $I R F$ intraretinal fluid, $S R F$ subretinal fluid.

\pm SD age of the 44 patients at baseline was $62 \pm 9$ years, and 22 $(50 \%)$ of the patients were female. The eyes received $7.9 \pm 3.5$ (mean $\pm \mathrm{SD}$ ) injections of bevacizumab during a mean $\pm \mathrm{SD}$ period of $10 \pm 4$ months prior to enrolment; 43 (90\%) of the 48 eyes received $\geq 6$ injections of bevacizumab prior to enrolling in the study. The baseline characteristics of the study cohort are summarised in Table 1.

Five patients withdrew from the study prior to completion. Two patients withdrew after the first visit (there are no CST data for these eyes), one patient withdrew after visit 3 (baseline and visit 3 CST were both $449 \mu \mathrm{m}$ ), one patient withdrew after visit 4 (CST was 432 and $497 \mu \mathrm{m}$ at baseline and visit 4 , respectively), and the fifth patient withdrew after visit 7 (CST was 882 and $626 \mu \mathrm{m}$ at baseline and visit 7, respectively).

None of the eyes received laser rescue treatment during the study. Three ocular adverse events were observed: one eye developed anterior uveitis during the follow-up period, one eye developed transiently elevated IOP, and one eye had a corneal aberration following an injection. No systemic adverse events were reported during the study.

\section{Anatomical outcome}

As shown in Fig. 1, average CST in the entire cohort decreased during the study from $468 \pm 131 \mu \mathrm{m}$ at baseline to $303 \pm 67 \mu \mathrm{m}$ at week $52(p=0.002$, Student's $t$-test $)$. This decrease in CST was already significant in week 4 (i.e., after the first aflibercept injection), in which CST was $395 \pm 83$ $\mu \mathrm{m}(p=0.008$ versus baseline, paired Student's $t$-test $)$. Although CST decreased in all cases comparing to baseline (data not shown), the decrease in CST was $<5 \%$ in three eyes.

\section{Treat-and-extend regimen (TER)}

A total of $20(46 \%)$ eyes met the TER criteria prior to week $20 ; 3(15 \%)$ of these 20 eyes started TER following the first injection (in week 4), 2 (10\%) started TER after the second injection (in week 8), $10(50 \%)$ started after the third injection (in week 12 ) and $5(25 \%)$ started after the forth injection (in week 16). The longest interval between injections was 10 weeks, which was in $5(25 \%)$ of the 20 eyes.

Three (15\%) of the 20 eyes that received TER required returning the injection interval back to the most recent fluidfree interval; in 2 of these eyes, the interval was reduced from 8 to 6 weeks; in 1 eye, the interval was reduced from 6 to 4 weeks. The mean \pm SD number of aflibercept injections during the study was $10.9 \pm 2$ in the eyes that were eligible for TER.

A total of 40 of the 48 eyes $(83 \%)$ had IRF at presentation (i.e. at baseline); by the end of the study (week 52), $31(72 \%)$ eyes had IRF ( $p=0.01$ versus baseline, chisquare test). In addition, ten eyes (21\%) had SRF at presentation, and none of the eyes had SRF at the end of the study ( $p=0.0001$ versus baseline, chi-square test).

As a control group for the primary end point, we included 13 eyes from 13 consecutive patients who were eligible for the TADI study after receiving 6 bevacizumab injections but continued with bevacizumab therapy for at least 12 additional months (i.e. did not enrol in the TADI study). In this control group, CST decreased during these 12 months from $477 \pm 139 \mu \mathrm{m}$ at baseline to $389 \pm 78$ micron ( $p=0.05$, paired Student's $t$-test). Although the baseline CST values were similar between the study group and the control group ( $468 \pm 131$ versus $477 \pm 139 \mu \mathrm{m}$, respectively; $p=0.4$, Student's $t$-test), the change in CST was significantly larger in the study group compared with the control group, with a decrease of $165 \pm 110 \mu \mathrm{m}$ versus $88 \pm 99 \mu \mathrm{m}$, respectively ( $p=0.001$, Student's $t$-test). The mean number of bevacizumab injections was similar between the study group and the control group $(11.6 \pm 1.7$ and $10.5 \pm 0.9$ injections, respectively; $(p=0.2$, Wilcoxonsigned rank). 


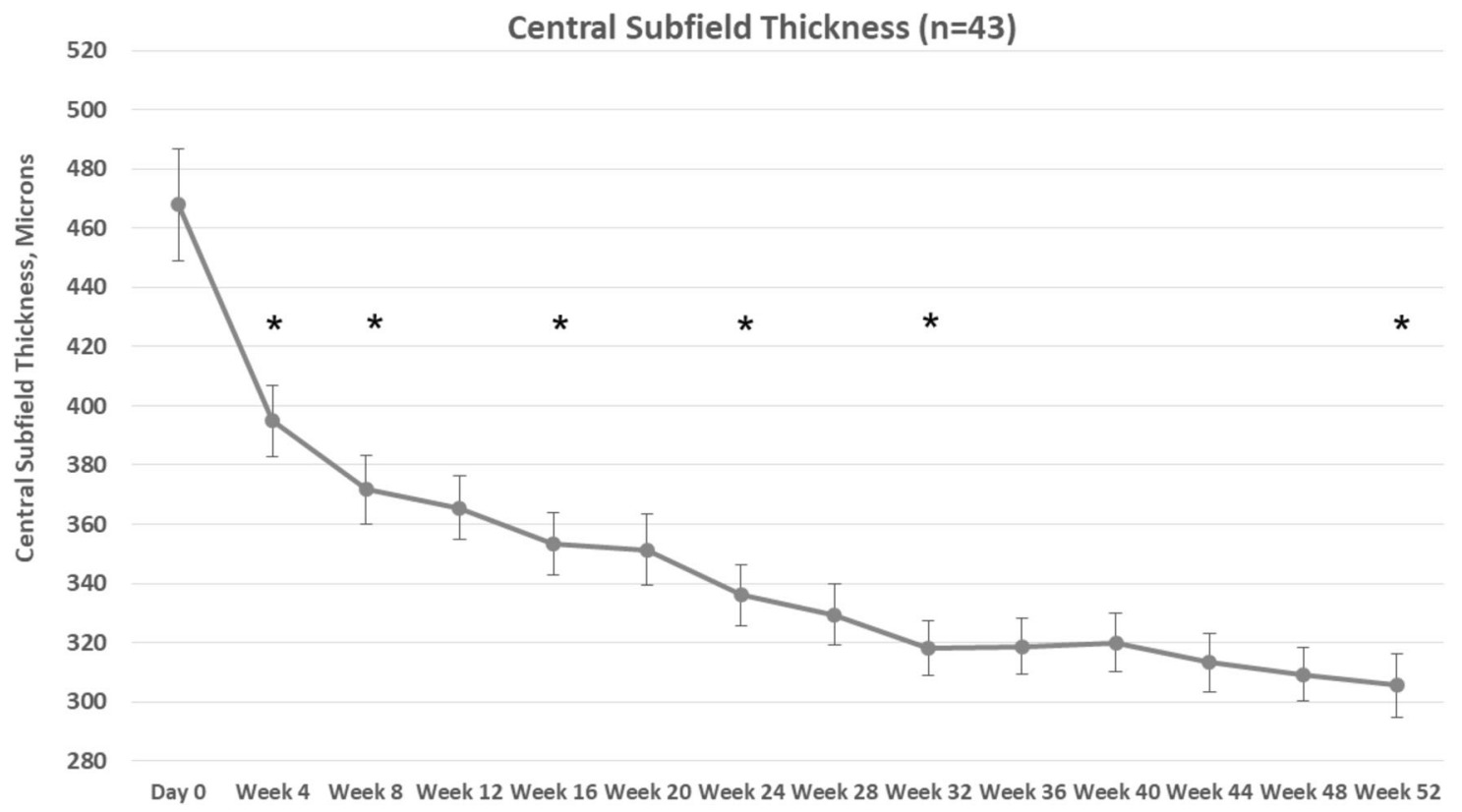

Fig. 1 Time course of central subfield macular thickness (CST) in 43 eyes measured using optical coherence tomography at baseline (day 0 ) and every 4 weeks throughout the study period. $* p<0.05$ versus baseline.

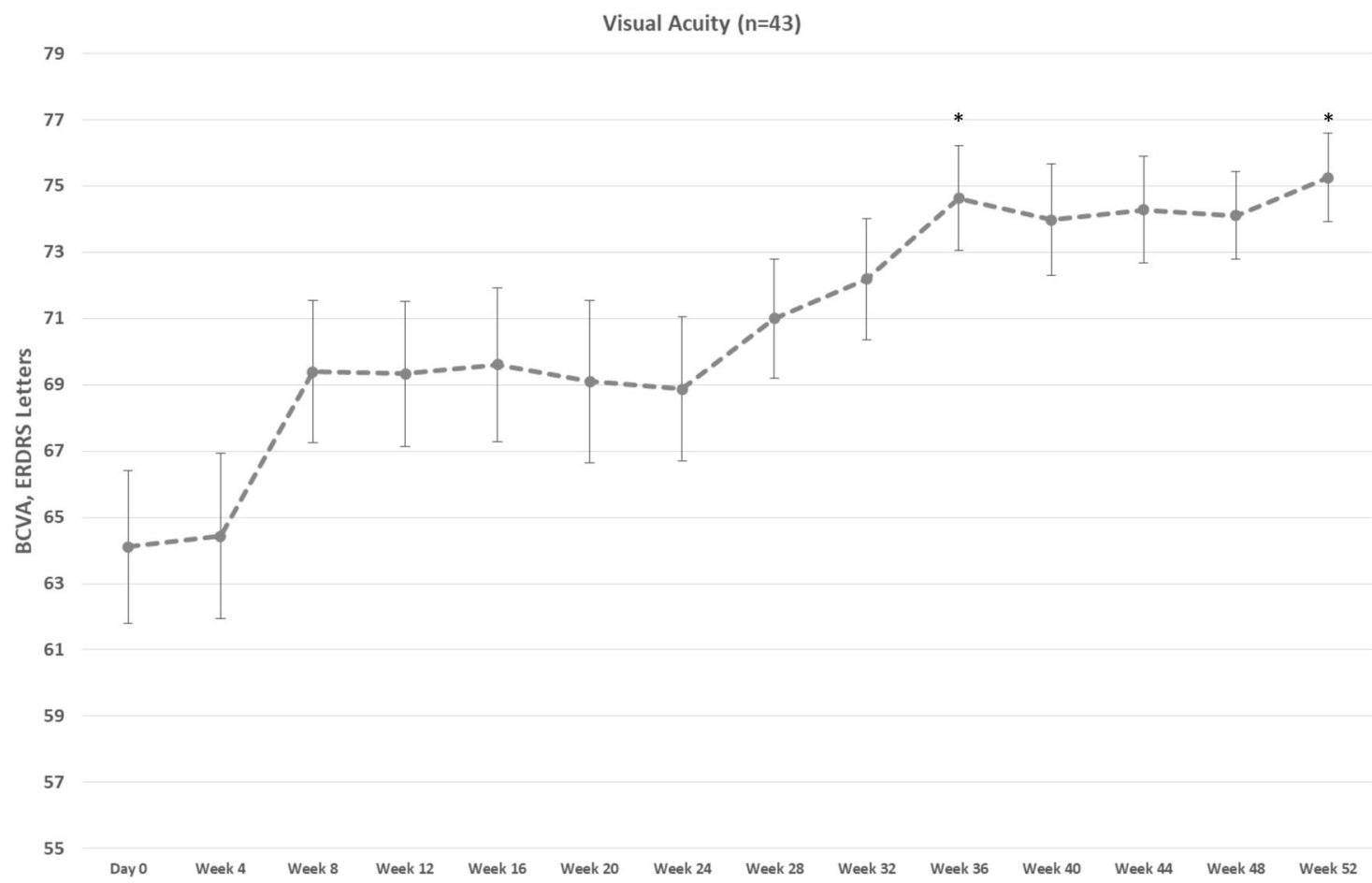

Fig. 2 Time course of best corrected visual acuity (BCVA) in ETDRS letters for 43 eyes measured at baseline (day 0 ) and every 4 weeks throughout the study period. ${ }^{*} p<0.05$ versus baseline.

\section{Visual acuity outcome}

As shown in Fig. 2, the mean \pm SD BCVA scores in the study group improved from $64 \pm 15$ letters at baseline to 75 \pm 8 letters at week $52(p=0.001$; paired Student's $t$-test $)$, with 34 (79\%) eyes improving by $\geq 1$ ETDRS letters and 20 (46\%) eyes improving by $\geq 10$ letters. In contrast, nine (21\%) eyes had a decrease of $\leq 10$ letters, and two (5\%) eyes had a decrease of $>10$ letters. Finally, BCVA in the historical control group (measured using an ETDRS chart in 


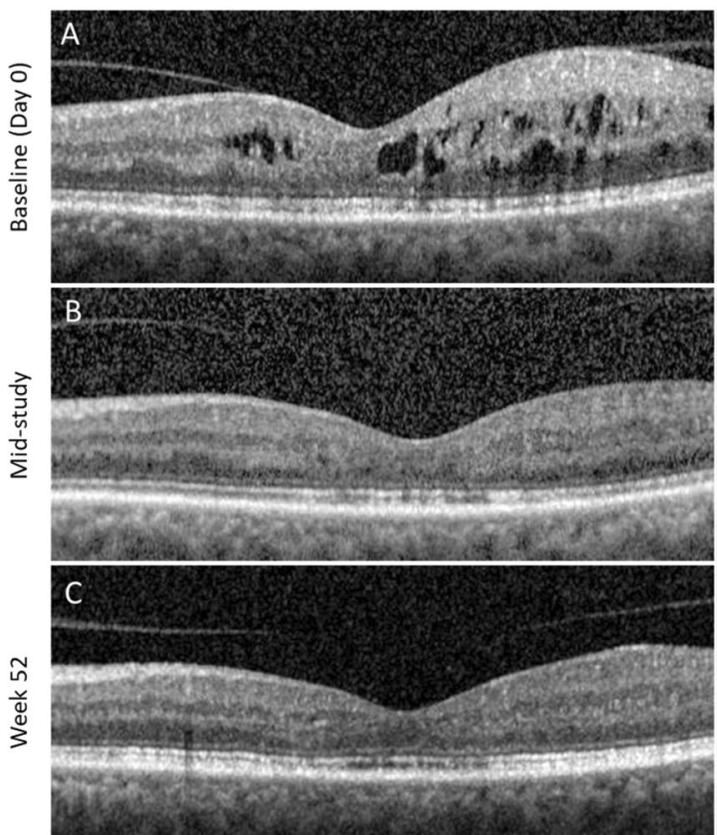

Fig. 3 Anatomical changes in SD-OCT during the study. a-c OCT scans of a 65-year-old male who presented with diabetic macular oedema (DMO) in the left eye following an incomplete response $(<10 \%$ reduction of central subfield macular thickness (CST)) to six injections of bevacizumab. Shown are OCT scans taken on day 0 (baseline; a), after 3 monthly injections of aflibercept (b; note the complete resolution of DMO, after which the patient was treated using the treat-and-extend regimen), and at the 52-week follow-up visit (c). a, c CST was 324 and $225 \mu \mathrm{m}$, respectively, and BCVA was 74 and 77

LogMAR) did not change significantly between baseline $(0.35 \pm 0.26 \operatorname{LogMAR})$ and after 12 months of treatment $(0.36 \pm 0.18 \operatorname{LogMAR} ; p=0.7$, paired Student's $t$-test $)$.

\section{Discussion}

The TADI study was a prospective, multicentre, openlabel, single-arm clinical trial designed to evaluate the efficacy of aflibercept as a second-line therapy for persistent DMO managed using an early TER without an initial obligatory monthly loading dose. All patients in the study had refractory DMO with persistent fluid despite 4-12 first-line bevacizumab injections prior to enroling in the study. Moreover, the entire cohort exhibited significant anatomical and functional improvement under aflibercept treatment, as illustrated in Fig. 3. At the 1-year follow-up visit, mean macular thickness had decreased by $>150 \mu \mathrm{m}$, and mean visual acuity had increased by 10 ETDRS letters compared with baseline. Forty percent of the eyes in the study met the criteria for TER treatment and began this treatment regimen prior to week 20 , but none was able to achieve a 12-week interval between injections.

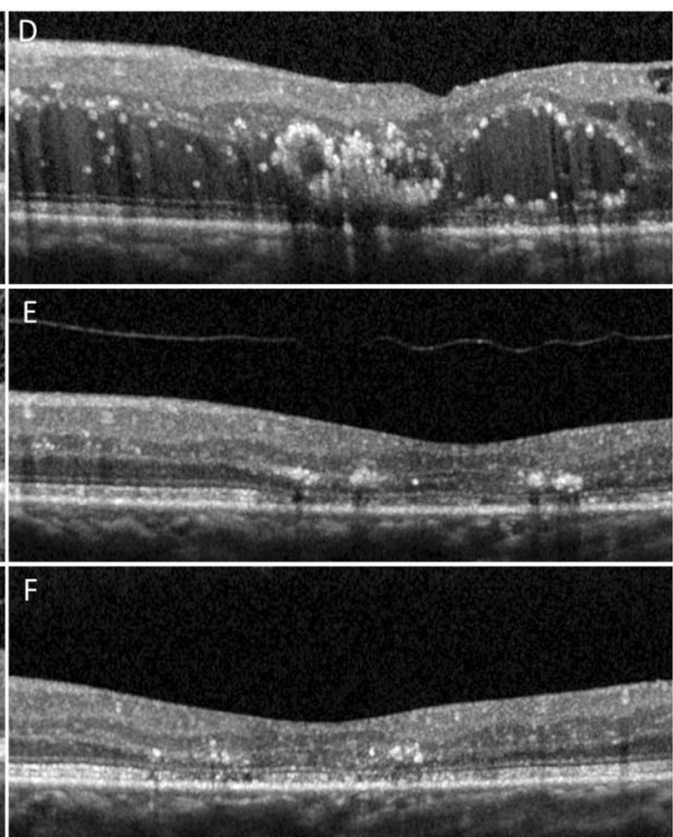

ETDRS letters, respectively. Over the course of 52 weeks, this patient received a total of 9 injections of aflibercept. d-f OCT scans of a 70year-old male who presented with refractory DMO in the left eye following six injections of bevacizumab with a complete lack of response (i.e., no change in CST from baseline). This patient was treated with monthly injections of aflibercept throughout the study period. Shown are OCT images at baseline (d), week 24 (e), and week 52 (f). d-f CST was 568, 268 and $213 \mu \mathrm{m}$, respectively; d, f BCVA was 70 and 77 ETDRS letters, respectively.

Several findings support the beneficial effect of switching to aflibercept in the study group. First, the patients received an average of 7.9 injections of bevacizumab before the switch. Second, a marked decrease in macular thickness was evident even after the first injection of aflibercept. Third, the decrease in macular thickness was larger in the eyes in the TADI group compared with the decrease seen in a historical control group. Forth, we observed a gradual improvement in thickness throughout the course of the study, along with a marked improvement in visual acuity following the switch to aflibercept.

The VIVID and VISTA studies demonstrated the efficacy of monthly and bi-monthly injections of aflibercept as a first-line therapy in DMO following a compulsory loading regimen of 5 monthly injections [8]. Moreover, the initial improvement in vision in these studies was stable over a long-term follow-up period [7, 8, 23]. In addition, the Protocol $\mathrm{T}$ trial conducted in the Diabetic Retinopathy Clinical Research Network revealed that aflibercept is superior to bevacizumab in both the first and second years of follow-up in eyes that presented with an initial visual acuity of 20/50 or worse [12, 13].

Although long-term analyses of data from Protocol T and Protocol I suggest that BCVA can improve even in the 
presence of persistent DMO provided that anti-VEGF treatment is continued, resolving the DMO can lead to an even better outcome in terms of visual acuity [34]. In addition, the prevalence of persistent DMO is lower-and visual outcome is better-in aflibercept-treated eyes compared with bevacizumab-treated eyes [35]. In our study, all of the included eyes lacked a sufficient response after an average of 7.9 bevacizumab injections before they were switched to aflibercept. In accordance with our findings, Do et al. reported a post-hoc analysis of the VISTA study in which both BCVA and macular thickness improved with aflibercept therapy in eyes that previously received antiVEGF (primarily bevacizumab) treatment [36].

The TREX-DME study found that using the TER approach with ranibizumab $0.3 \mathrm{mg}$ as the first-line therapy yield significant improvement in visual acuity and central retinal thickness in eyes with DMO. In the TREX-DME study, all eyes received 4 monthly ranibizumab injections and were placed on a TER regimen when CST reached $\leq 325 \mu \mathrm{m}$ [31]. In our study, the TER protocol differed in several aspects. First, we initiated the TER regimen only after DMO was completely resolved, without including a mandatory monthly loading dose. Second, we included patients with CST $\geq 300 \mu \mathrm{m}$. Third, we did not a priori randomise the eyes into monthly and TER groups; rather, we initiated the TER regiment in any eye in which complete resolution of DMO was achieved. Interestingly, we observed a mean decrease in CST of $60 \mu \mathrm{m}$ even after the first injection of aflibercept, and $50 \%$ of the patients who were placed on TER therapy started this regimen following their third injection of aflibercept or earlier.

Bahrami and colleagues recently reported a prospective study in which intravitreal aflibercept was injected in 41 eyes in 41 patients with persistent DMO despite first-line bevacizumab therapy [37]. In their study, five injections were administered every 4 weeks, with subsequent injections administered every 8 weeks. At 48 weeks, BCVA had improved by $3.9 \pm 7.0$ ETDRS letters. Nine of the 41 patients were treated simultaneously with bevacizumab in fellow eyes, and these patients had a similar improvement in visual acuity in both eyes during the study [37]. The superior visual outcome for eyes in the TADI study compared with Bahrami el al.'s findings may be explained-at least in part-by the fact the TADI patients received fewer bevacizumab injections before the switch (7.9 injections on average compared with a median of 12 injections in the study by Bahrami el al.). Other potential explanations may include other differences in the patient populations and/or differences in the treatment protocol. Specifically, in the TADI study more than half of the patients required monthly therapy for at least 12 months following the switch from bevacizumab to aflibercept. In support of this possible explanation, Bahrami et al. found a decrease in macular thickness while patients received monthly injections; under the bi-monthly regimen (starting from the 6th injection), there appeared to be no change in macular thickness. In contrast, the patients in the TADI study had a persistent decrease in macular thickness throughout the study period, which may reflect the fact that many of the eyes in our study required monthly injections throughout the 12-month study period.

The TADI trial had several caveats that warrant discussion. First, our study included a relatively small number of eyes; however, despite the small sample size, we observed a significant improvement in both vision and central thickness following the switch to aflibercept. Second, we did not include a control group in which the eyes were not treated with aflibercept; however, we did include historical data from a small group of eyes that did not switch to aflibercept despite meeting the inclusion criteria used for the TADI study; this group had worse outcome compared with the TADI group. It is important to note that the historical control group received a mean of 10.5 bevacizumab injections versus a mean of 11.6 aflibercept injections in the TADI group during the 12-month study period. Nevertheless, this difference was not statistically significant and is unlikely to account for the average difference of $77 \mu \mathrm{m}$ in CST change between the groups. In support of this notion, the 11th aflibercept injection in the TADI group resulted in a mean decrease in CST of only $\sim 5 \mu \mathrm{m}$. Finally, the longest interval between injections in the TER group was 10 weeks (not 12 weeks). It is also important to note that even though significant improvement was achieved, approximately half of the eyes in the study could not be extended in order to maintain this improvement. This might suggest that the eyes in the TADI study required more intensive treatment compared with eyes that are treatment-naïve at the start of aflibercept therapy. Nevertheless, even in previously treated eyes, monthly treatment may often be avoided, and the treatment interval could be 8 weeks or longer.

In conclusion, we report the results from the first prospective trial designed to assess the efficacy of using aflibercept as a second-line treatment with a modified TER for treating refractory DMO following first-line bevacizumab therapy. We found that switching to aflibercept after initial therapy with bevacizumab for eyes with refractory DMO markedly decreased macular thickness and improved visual acuity within one year. The modified TER using aflibercept without a mandatory loading dose enabled us to extend the treatment interval to longer than 4 weeks in approximately half of the eyes. Future studies should examine the putative benefits of second-line anti-VEGF therapies in eyes following an incomplete response to bevacizumab. Such an approach will likely facilitate the development of practical treatment protocols designed using evidencebased data. 


\section{Summary}

\section{What was known before}

- Aflibercept is an efficient first-line anti-VEGF treatment for diabetic macular oedema.

\section{What this study adds}

- Aflibercept is an effective second-line treatment for patients with an incomplete response to previous bevacizumab for DMO, using the modified TER without an obligatory loading phase.

Acknowledgements We would like to thank the clinical trials unit of the department of ophthalmology at the Hadassah-Hebrew University Medical Centre for managing and coordinating between participating centres.

Author contributions LT and IC provided a substantial contribution to the conception of the work, analysis and interpretation of data, drafting of the manuscript, and providing the final approval for the submitted manuscript. RE, YB, HK, RP, TJ, JL, JH, OS, SS and MG contributed to the data collection, analysis and interpretation of data and gave the final approval of the submitted manuscript.

Funding Bayer Israel funded the study and provided the study medication. The study was designed, conceived and conducted, and analysed independently by the authors; Bayer Israel had no control over the data or the decision to publish.

\section{Compliance with ethical standards}

Conflict of interest The study was supported by Bayer Israel.

Ethics approval The study protocol was approved by the Institutional Review Board of the Hadassah-Hebrew University Medical Center and all other participating sites.

Patient consent Obtained.

Publisher's note Springer Nature remains neutral with regard to jurisdictional claims in published maps and institutional affiliations.

\section{References}

1. Klein R, Klein BE, Moss SE, Cruickshanks KJ. The wisconsin epidemiologic study of diabetic retinopathy. XV. The long-term incidence of macular edema. Ophthalmology. 1995;102:7-16.

2. Varma R, Bressler NM, Doan QV, Gleeson M, Danese M, Bower $\mathrm{JK}$, et al. Prevalence of and risk factors for diabetic macular edema in the United States. JAMA Ophthalmol. 2014;132: 1334-40.

3. Yau JW, Rogers SL, Kawasaki R, Lamoureux EL, Kowalski JW, Bek T, et al. Global prevalence and major risk factors of diabetic retinopathy. Diabetes Care. 2012;35:556-64.
4. Anon. A randomized trial comparing intravitreal triamcinolone acetonide and focal/grid photocoagulation for diabetic macular edema. Ophthalmology. 2008;115:1447-9. 1449 e1-10.

5. Elman MJ, Aiello LP, Beck RW, Bressler NM, Bressler SB, Edwards AR, et al. Randomized trial evaluating ranibizumab plus prompt or deferred laser or triamcinolone plus prompt laser for diabetic macular edema. Ophthalmology. 2010;117:1064-77. e35.

6. Elman MJ, Bressler NM, Qin H, Beck RW, Ferris FL 3rd, Friedman SM, et al. Expanded 2-year follow-up of ranibizumab plus prompt or deferred laser or triamcinolone plus prompt laser for diabetic macular edema. Ophthalmology. 2011;118:609-14.

7. Brown DM, Schmidt-Erfurth U, Do DV, Holz FG, Boyer DS, Midena E, et al. Intravitreal aflibercept for diabetic macular edema: 100-week results from the VISTA and VIVID studies. Ophthalmology. 2015;122:2044-52.

8. Korobelnik JF, Do DV, Schmidt-Erfurth U, Boyer DS, Holz FG, Heier JS, et al. Intravitreal aflibercept for diabetic macular edema. Ophthalmology. 2014;121:2247-54.

9. Mitchell P, Bandello F, Schmidt-Erfurth U, Lang GE, Massin P, Schlingemann RO, et al. The RESTORE study: ranibizumab monotherapy or combined with laser versus laser monotherapy for diabetic macular edema. Ophthalmology. 2011;118:615-25.

10. Nguyen QD, Brown DM, Marcus DM, Boyer DS, Patel S, Feiner $\mathrm{L}$, et al. Ranibizumab for diabetic macular edema: results from 2 phase III randomized trials: RISE and RIDE. Ophthalmology. 2012;119:789-801.

11. Wells JA, Glassman AR, Ayala AR, Jampol LM, Aiello LP, Antoszyk AN, et al. Aflibercept, bevacizumab, or ranibizumab for diabetic macular edema. N. Engl J Med. 2015;372:1193-203.

12. Cai S, Bressler NMAflibercept. bevacizumab or ranibizumab for diabetic macular oedema. Curr Opin Ophthalmol 2017;28:636-43. http://insights.ovid.com/crossref?an $=00055735-201711000-00016$.

13. Wells JA, Glassman AR, Ayala AR, Jampol LM, Bressler NM, Bressler SB, et al. Aflibercept, bevacizumab, or ranibizumab for diabetic macular edema: two-year results from a comparative effectiveness randomized clinical trial. Ophthalmology. 2016;123: 1351-9.

14. Nepomuceno AB, Takaki E, Paes de Almeida FP, Peroni R, Cardillo JA, Siqueira RC, et al. A prospective randomized trial of intravitreal bevacizumab versus ranibizumab for the management of diabetic macular edema. Am J Ophthalmol. 2013;156:502-10. e2.

15. Maturi RK, Glassman AR, Liu D, Beck RW, Bhavsar AR, Bressler NM, et al. Effect of adding dexamethasone to continued ranibizumab treatment in patients with persistent diabetic macular edema: a drcr network phase 2 randomized clinical trial. JAMA Ophthalmol. 2017;136:29-38.

16. Ashraf M, Souka A, Adelman R, Forster SH. Aflibercept in diabetic macular edema: evaluating efficacy as a primary and secondary therapeutic option. Eye 2016;30:1531-41.

17. Demircan A, Alkin Z, Yesilkaya C, Demir G, Kemer B. Comparison of intravitreal aflibercept and ranibizumab following initial treatment with ranibizumab in persistent diabetic macular edema. J Ophthalmol. 2018;2018:4171628.

18. Herbaut A, Fajnkuchen F, Qu-Knafo L, Nghiem-Buffet S, Bodaghi B, Giocanti-Auregan A. Switching to aflibercept in diabetic macular edema not responding to ranibizumab and/or intravitreal dexamethasone implant. J Ophthalmol. 2017;2017:8035013.

19. Klein KA, Cleary TS, Reichel E. Effect of intravitreal aflibercept on recalcitrant diabetic macular edema. Int J Retin Vitr. 2017;3:16.

20. McCloskey CF, Mongan AM, Chetty S, McAteer DMJ, Quinn SM. Aflibercept in diabetic macular oedema previously refractory to standard intravitreal therapy: an irish retrospective study. Ophthalmol Ther 2018;7:173-83.

21. Mira F, Paulo M, Henriques F, Figueira J. Switch to aflibercept in diabetic macular edema patients unresponsive to previous antiVEGF therapy. J Ophthalmol. 2017;2017:5632634. 
22. Ehrlich R, Pokroy R, Segal O, Goldstein M, Pollack A, Hanhart J, et al. Diabetic macular edema treated with ranibizumab following bevacizumab failure in Israel (DERBI study). Eur J Ophthalmol. 2018;29:229-33.

23. Heier JS, Korobelnik JF, Brown DM, Schmidt-Erfurth U, Do DV, Midena E, et al. Intravitreal aflibercept for diabetic macular edema: 148-week results from the VISTA and VIVID studies. Ophthalmology. 2016;123:2376-85.

24. Sun JK, Jampol LM. The diabetic retinopathy clinical research network (DRCR.net) and its contributions to the treatment of diabetic retinopathy. Ophthalmic Res. 2019;62:225-30.

25. Spaide R. Ranibizumab according to need: a treatment for agerelated macular degeneration. Am J Ophthalmol. 2007;143: 679-80.

26. Gupta OP, Shienbaum G, Patel AH, Fecarotta C, Kaiser RS, Regillo CD. A treat and extend regimen using ranibizumab for neovascular age-related macular degeneration clinical and economic impact. Ophthalmology. 2010;117:2134-40.

27. Abedi F, Wickremasinghe S, Islam AF, Inglis KM, Guymer RH. Anti-VEGF treatment in neovascular age-related macular degeneration: a treat-and-extend protocol over 2 years. Retina. 2014;34: 1531-8.

28. Rayess N, Houston SK 3rd, Gupta OP, Ho AC, Regillo CD. Treatment outcomes after 3 years in neovascular age-related macular degeneration using a treat-and-extend regimen. Am J Ophthalmol. 2015;159:3-8. e1.

29. Berg K, Pedersen TR, Sandvik L, Bragadottir R. Comparison of ranibizumab and bevacizumab for neovascular age-related macular degeneration according to LUCAS treat-and-extend protocol. Ophthalmology. 2015;122:146-52.

30. Payne JF, Wykoff CC, Clark WL, Bruce BB, Boyer DS, Brown DM. Randomized trial of treat and extend ranibizumab with and without navigated laser for diabetic macular edema: TREX-DME
1 year outcomes. Ophthalmology. 2017;124:74-81. https://doi. org/10.1016/j.ophtha.2016.09.021.

31. Prünte C, Fajnkuchen F, Mahmood S, Ricci F, Hatz K, Studnička $\mathrm{J}$, et al. Ranibizumab $0.5 \mathrm{mg}$ treat-and-extend regimen for diabetic macular oedema: The RETAIN study. $\mathrm{Br} \mathrm{J}$ Ophthalmol. 2016;100:787-95.

32. Payne JF, Wykoff CC, Clark WL, Bruce BB, Boyer DS, Brown DM. Randomized trial of treat and extend ranibizumab with and without navigated laser versus monthly dosing for diabetic macular edema: TREX-DME 2-year outcomes. Am J Ophthalmol. 2019;202:91-9.

33. Grover S, Murthy RK, Brar VS, Chalam KV. Comparison of retinal thickness in normal eyes using Stratus and Spectralis optical coherence tomography. Invest Ophthalmol Vis Sci. 2010; 51:2644-7.

34. Krick TW, Bressler NM. Recent clinically relevant highlights from the diabetic retinopathy clinical research network. Curr Opin Ophthalmol 2018;29:199-205.

35. Bressler NM, Beaulieu WT, Glassman AR, Blinder KJ, Bressler SB, Jampol LM, et al. Persistent macular thickening following intravitreous aflibercept, bevacizumab, or ranibizumab for centralinvolved diabetic macular edema with vision impairment: a secondary analysis of a randomized clinical trial. JAMA Ophthalmol. 2018;136:257-69.

36. Do DV, Nguyen QD, Vitti R, Berliner AJ, Gibson A, Saroj N, et al. Intravitreal aflibercept injection in diabetic macular edema patients with and without prior anti-vascular endothelial growth factor treatment outcomes from the phase 3 program. Ophthalmology. 2016;123:850-7. https://doi.org/10.1016/j.ophtha.2015. 11.008 .

37. Bahrami B, Hong T, Schlub TE, Chang AA. Aflibercept for persistent diabetic macular edema: forty-eight-week outcomes. Retina. 2019;39:61-8. 